\title{
A Review of Event-Related Potential (ERP) Components Employed in Mental Arithmetic Processing Studies
}

\section{E.T. Muluh*}

Faculty of Engineering, Cape Peninsula University of Technology, Bellville campus, Simphony way 7535, South Africa

\begin{abstract}
This review focuses on studies utilizing early, late and slow-wave components of event-related potentials (ERPs) in investigating two mental arithmetic processing (MAP) phenomenons - problem-size and arithmetic-operation effects. While MAP has been associated primarily with slow-wave components of ERPs, inconsistent findings are found in the literature on the sensitivity of early and some late components. In an attempt to theoretically integrate the results of reviewed studies, it is proposed that the amplitude of early and late ERP components are potential indices for measuring the effect of manipulating the problem-size and/or arithmeticoperation during MAP. This conclusion will help in providing more insight into the strategies employed in MAP by the human brain.
\end{abstract}

Keywords: Event-related potentials; Arithmetic-operation effect; Problem-size effect; Mental arithmetic processing

\section{Introduction}

Arithmetic from the Greek word aı $\theta \mu$ ó $\zeta$ means number and is the oldest and simplest branch of mathematics with four basic operations - addition, subtraction, multiplication and division. Despite the apparent ease with which we decide mentally that $5-2$ is $3,4 \times 6$ is 24 , $5+3$ is $8,24 \div 6$ is 4 and so on, the literature attest to the complexity of mental processing involved in these seemingly elementary tasks $[1,2]$. Although mental arithmetic is a relatively circumscribed domain with explicit rules, facts, and principles, it still shares the processing complexity of other cognitive tasks and appears to use the same mental structures and memory systems [3].

In fact, the performance of this complex cognitive task by the human brain requires the management of a variety of mental processes and information sources. This complexity has led to the investigation of several mental arithmetic processing (MAP) effects. Two of them the problem-size effect (PSE) - a situation where people are slower and less accurate in solving problems with numerically large digits and answers than solving problems with numerically small digits and answers $[4,5]$ and the arithmetic-operation effect (AOE) - the dissociation observed during processing of addition, division, multiplication and subtraction operations [6,7]. These two effects are currently enjoying a lot of research interest in cognitive arithmetic. As a result of this, several techniques have been employed in studying these effects in numerical cognition.

In the past, numerical cognition has been extensively investigated using mostly behavioral methods. But over the past two decades, psychophysiological measures such as event-related potentials (ERPs) and functional Magnetic Resonance Imaging (fMRI) have provided mounting evidence on the neurobiological basis of MAP. Using these techniques and others, attempts have been made to answer the main question in cognitive arithmetic - how do people do arithmetic in their brain? This is an interesting question for cognitive science because our ability (or inability) in mental arithmetic points to the limitations of the human mind.

Several studies have utilized several ERP components in investigating PSE and AOE in MAP. Some of these studies have suggested that ERPs wave forms reflected a complex of higher order brain functions involved in MAP, including the processes of identifying numerals, performing mathematical calculations and memorizing results [8]. These suggestions are consistence with case and behavioral studies [9]. In fact, Sandrini and colleagues in their study concluded that people (a) process numerical information (i.e., perceive, comprehend, and produce numbers); (b) process operational signs indicating specific calculations to be performed; (c) access arithmetic table facts (e.g. $4 \mathrm{x}$ $8=32 ; 8+4=12$ ); (d) execute calculation procedures that specify the sequence of steps to be carried out in solving multi-digit problems; and (f ) understand the arithmetic operations and principles (conceptual knowledge) during MAP.

Appreciable progress has been made in the last two decades in employing ERPs in MAP studies to investigate PSE and AOE. However, differences often in experimental design, methodology, manipulation of problem-size, arithmetic-operation, analysis technique(s) among others have been observed to characterize ERP studies investigating these two effects. While it is likely that a number of these differences and others not mentioned may influence modulation of ERP components during MAP, a considerable number of these differences have not been resolved properly.

Firstly, component modulation by AOE x PSE interaction has been confounded in several MAP studies. That is, several studies have interpreted this interaction as PSE or AOE alone [10-15]. Secondly, the range of small and large size problems employed in studies varies from 2 - 21 for small size and 6 - 89 for large size problems with no agreement on the number of levels on which the size should be manipulated. Some authors have used three levels comprising of small, medium and large size problems [16-18] while others have used two levels only - small and large size problems [19-22,15,23]. Thirdly, the manipulation of arithmetic-operation has often been compounded in a

*Corresponding author: E.T. Muluh, Faculty of Engineering, Cape Peninsula University of Technology, Bellville campus, Simphony way 7535 , South Africa, E-mail: muluhe@cput.ac.za

Received November 06, 2011; Accepted November 25, 2011; Published December 10, 2011

Citation: Muluh ET (2011)A Review of Event-Related Potential (ERP) Components Employed in Mental Arithmetic Processing Studies. J Neurol Neurophysiol S6. doi:10.4172/2155-9562.S6-001

Copyright: @ 2011 Muluh ET. This is an open-access article distributed under the terms of the Creative Commons Attribution License, which permits unrestricted use, distribution, and reproduction in any medium, provided the original author and source are credited. 
single task involving two or more arithmetic-operations. For example, consecutive addition or subtraction as in the studies of $\mathrm{Nu}^{\prime} \mathrm{n}^{\sim} \mathrm{ez}-\mathrm{Pen}$ a et al. $[13,14]$ with the studies of Zhou et al. $[15,24,23]$ as exceptions. Fourthly, the range of filters used in extracting ERP data vary across studies from $0.01 \mathrm{~Hz}$ to $100 \mathrm{~Hz}$. Fifthly, even though PSE modulation of ERP components has been shown not to be due to differences in the activation of the correct results only, most studies have often not investigated question-locked ERP components during MAP. In fact, different solution strategies have been shown to be contributing factors for PSE which may start immediately after the presentation of the question-stimuli $[25,26]$. These variations may mask several MAP processes leading to potential differences in findings across studies and possible misinterpretation of effects. Thus suitable manipulation of experimental variables such as problem-size and arithmetic-operation together with a proper ERP frequency band separation of early, late and slow-wave components may show real amplitude and latency modulation by AOE, PSE and AOE x PSE during MAP.

As already pointed out, studies have differentially associated several MAP effects with early, late and slow-wave components of ERPs [10,12,19,20,27,8,25,21,13,14,18]. Almost all studies employing this technique have used a delayed verification task. This approach renders behavioral data collected from these studies not very reliable as a measure of PSE or AOE modulation of latency. Thus, in the present review focus is more on the amplitude and to a lesser degree the latency of ERP components. Early, late and slow-waves components of ERP have been employed in several of these studies with no agreement on where these components post-question stimulus starts and ends. Thus, the usage of early, late and slow-wave in the present review may not refer to a unique interval across studies.

\section{Problem-size and arithmetic-operation effects in early ERP components}

Mental arithmetic processing has been associated primarily with slow-wave components of ERPs as already mentioned [10-12,8,13,14]. Regarding early and some late components, inconsistent findings are recorded in the literature on their sensitivity or insensitivity to MAP effects. Early components have been argued to be linked with calculation as numerals/operation signs have to be recognized/comprehended during MAP [28]. Some researchers in fact have argued in favour of modulation of these components by MAP effects such as PSE and/or AOE $[20,21,23]$. However, other studies have shown no modulation of these components as a result of manipulating the problem-size [13].

Early ERP components such as P1 and P2 (positive going peaks around $100 \mathrm{~ms}$ and $200 \mathrm{~ms}$ respectively post stimulus presentation) are elicited by mental arithmetic processing as well as other mental activities such as counting meaningless patterns $[20,8,21,13]$. These components in the past have generally been considered to reflect attention to digit-patterns and the physical identification of numbers and their meaning $[8,21]$. However, some studies have associating the P2 component with PSE [20] and recently AOE [23].

In the study of Iguchi and Hashimoto, three tasks - (1) addition of numbers between 3 and 9 successively, (2) counting the number of presented digits and (3) counting meaning- less patterns were performed. The addition sign operation $(+)$ was not employed during problem presentation in this study. Event-relate potentials were filtered into low $(0.0-3 \mathrm{~Hz})$, high $(3-15 \mathrm{~Hz})$ and original $(0-15$ $\mathrm{Hz})$ ) ERP frequency bands and analyzed for sequential information processing during MAP. An N120-P180-N220 complex that advanced in latency in the left frontal, central and parietal regions was observed. This complex was associated with attention to digit-patterns during addition. The P180 part of this complex was identified not to be affected by MAP. The P180 in that study corresponded to the P1 component whose amplitude was shown later not to be modulated by problem-size manipulation (e.g. in the study of Sz"ucs and Cs'epe [21]).

Szu"cs and Cs'epe [21] tasked their subjects to add a visually presented Arabic number to a preceding (1) Arabic, (2) written number word, and (3) an acoustically presented number word. Subjects were required to decide whether a presented answer is correct or incorrect by a press of button. The operation sign $(+)$ was employed during problem presentation by these author. ERPs filtered in the $0.1-30 \mathrm{~Hz}$ frequency-band where analyzed for abstract amodal and modalitydependent presentation of numbers. Large (resulting sum between 11 and 18) and small (resulting sum between 3 and 10) size problems were used in that study. These authors attributed the latency effects to perceptual processes such as the fast extraction of number meaning which probably was different in different modalities. A PSE x modality interaction effect was also observed in that study. Amplitude differences were not significant in the three modalities employed by Szu"cs and Cs'epe. The latency of the P1 component was significantly modulation at parietal electrodes ( $\mathrm{P} 3, \mathrm{Pz}$, and $\mathrm{P} 4)$ in the three conditions. Latency effect was attributed to quantity representation.

More recently, Muluh et al. [23] in their study presented four blocks of (1) addition (117 large and 117 small problems), (2) division (117 large and 117 small problems), multiplication (117 large and 117 small problems), and subtraction (117 large and 117 small problems) to their subjects. Problems were presented in a standard format followed by three answer choices. The four basic arithmetic operation signs (+, $\therefore, \mathrm{X},-$ ) were employed in that study. Electroencephalography recorded was filtered in the 0.1 to $12 \mathrm{~Hz}$ band and analyzed for AOE. Early ERP components were modulated by manipulating the arithmeticoperation. Modulated was observed as early as $100 \mathrm{~ms}$ post-question presentation (in high frequency band). AOE was very pronounced in large-size problems in that study.

For the P2 component, N'ũnez-Pe na et al. [13] presented their subjects with (1) consec- utive addition or (2) subtraction of 2, 3 or 4 to or from the previous number constructed from a sequence of seven Arabic numbers. The three increments or reductions were selected so as to manipulate the problem-size $(2,3$ considered small size addition or subtraction and 4 large size). The seventh number presented completed the sequence either correctly or incorrectly and subjects were required to indicate this by a motor response. The operation signs + and - were not employed during problem presentation in that study. Event-related potentials were filtered into $0.05-30 \mathrm{~Hz}$ frequency band. Eventrelated potentials for the sixth number in the addition and subtraction sequences were analyzed for PSE. However, problem-size modulation failed to reveal any significant P2 amplitude or latency modulation in serial addition or subtraction. This observation was associated with the identification of stimulus which included the encoding phase for classical ERP pattern connected to visual stimuli recognition. Amplitude "size" of this component has also been associated with "paying" attention to digit patterns by subjects during addition (e.g., Iguchi and Hashimoto [8]). No amplitude or latency differences between conditions (addition or subtraction of 2, 3 or 4) were found up to approximately $250 \mathrm{~ms}$ post-stimulus by $\mathrm{N}^{\prime} \mathrm{u}^{\sim}$ nez-Pe $\sim$ na et al. [13].

Interestingly, Kong et al. [20] in their study presented sequential addition of visually presented double and single-digit numbers together 
with ' + ' sign to their subjects. Problems were separated into difficult (first operand - a two-digit number between 13 and 87 added to second operand- a one-digit number between 7 - 9) and easy (first operand - a two-digit number between 13 and 87 added to second operand - a onedigit number between 1 - 2) problems. Event-related potentials were filtered into $0.05-100 \mathrm{~Hz}$ and analyzed for PSE. Kong et al. [20] argued in favour of PSE modulating the amplitude of P2. In fact, significant amplitude differences at electrode F3 (difficult problems being more positive compared to easy ones) was observed. A significant latency effects at electrode F7 (difficult problems having longer latencies compared to easy ones) was also observed. Latency effects by PSE at electrode F4 have been associated with P3a effects by Kong et al. [20], a variant $\mathrm{P} 3 \mathrm{~b}$. On the other hand, amplitude enhancement (of possibly P3a) in addition and counting tasks at frontal and temporal regions has been interpreted as recognition of numeric meaning of digits (Iguchi and Hashimoto, 2000).

Also, Sz"ucs and Cs'epe [21] found a PSE x modality interaction effect in their study. The amplitude of $\mathrm{P} 2$ was significantly affected when frontal (F3, Fz, F4) and central $(\mathrm{C} 3, \mathrm{Cz}, \mathrm{C} 4)$ electrodes where analyzed for the PSE in the three modalities (Arabic, written number word and acoustically presented number word) by these authors. Higher amplitude were recorded for large size problems in Arabic and acoustically presented problems compared to small size problems in written number word. Latency was not affected by modality presentation. Amplitude effects were attributed to perception processes including the fast extraction of the meaning of presented numbers by these authors. Muluh et al. [23] recently also recorded significant AOE of the P2 component. These findings seem to suggest that early ERP components were modulated by other effects beside the PSE (e.g. AOE, AOE x PSE interaction).

\section{Problem-size and arithmetic-operation effects in Late ERP components}

As already mentioned above and similar to early components, late ERPcomponents have been shown to be enhance [8] modulated $[19,15,23]$ as well as not modulated $[16,17,20]$ during MAP. Late ERP components such as $\mathrm{P} 3 \mathrm{~b}$ and N3 (positive and negative going peaks respectively around $300 \mathrm{~ms}$ post stimulus presentation) are also generally associated with classification of stimulus $[16,17,8,13]$ or retrieval of MAP facts $[19,15]$.

For the P3b component, amplitude and latency modulation by AOE $\mathrm{x}$ PSE have resulted in diverse findings $[10,12,16,19,20,8,22,21,15]$. For instance, amplitude and latency at central and parietal elec- trode locations have been shown not to be significantly modulation by PSE $[16,17,20,21]$. Pauli et al. $[16,17]$ presented subjects with single-digit multiplication problems separated into easy (product result be- tween 10 - 21), moderate (product result between $24-40$ ) and difficult (product result between $42-72$ ) problems. The operation sign ( $\mathrm{x}$ ) was not employed during problem presentation in these studies. Eventrelated potentials were filtered into $0.0796-35 \mathrm{~Hz}$ frequency-band and analyzed for the four conditions (easy, moderate, and difficult). In the study of 1994, Pauli and colleagues observed a late positivity with peak at $300 \mathrm{~ms}$ that seemed a 'P3b-like' (according to these authors) component that was not significant in amplitude or latency. These authors were reluctant to call it a P3b because of lack of a clear parietal maximum. The component was associated with general informationprocessing processes that were not specific to mental arithmetic. Later in the 1996 study, they observed a similar late positive component with a distinct peak at about $300 \mathrm{~ms}$ that was significantly affected by practice and not problem difficulty at frontal and central electrode locations.

In a four-tasks study, Ruchkin et al. [10] presented to their subjects (1) memorize - corresponding to memorizing the rightmost digit of a presented three-digit number (considered to be an easy concept), (2) subtraction - corresponding to computing the magnitude of the difference between the right-most and center digit of a presented three-digit number (considered to be a less easy concept), (3) division - corresponding to dividing the right-most digit by the number composed by the other two-digits of a presented three-digit number (considered to be a difficult concept), and (4) discriminate - corresponding to discriminating between an intact number (considered to be an easy perception) and degraded number (considered to be a difficult perception). Operation signs $\div$ and - were not employed during problem presentation in this study. Eventrelated potentials filtered bellow $3.4 \mathrm{~Hz}$ were analyzed to differentiate conceptual (arithmetic) and perceptual (pattern recognition) difficulty. In a similar experimental set up, R"osler and Heil [12] asked their subjects to (1) memorize the right-most digit of a presented threedigit number (considered to be easy), (2) compute the magnitude of the difference between the right-most and center-digit (considered to be less easy), and (3) compute the reminder from the division of the right-most digit into the number composed by the other two-digits of the presented three-digit number (considered to be difficult). Subjects were required to give a response at the end of each task. Operation signs $(\div,-)$ were also not employed during problem presentation. Event-related potentials filtered $0.016-5.0 \mathrm{~Hz}$ were analyzed for the three conditions. Significant amplitude modulation of $\mathrm{P} 3 \mathrm{~b}$ at parietal electrodes by AOE X PSE interaction was observed by these authors $[10,12]$. However, these studies did not explicitly refer to the effect as an interaction. The observed amplitude effect was associated with recognition of task and adjustment of sets and or resources necessary for performing calculation. Interestingly, the ERP frequency band employed by these authors corresponds to the ERP low frequency band considered by Iguchi and Hashimoto [8]. It is possible that this might have enhanced the observed effect as P3b is considered a low frequency band component (e.g., Iguchi and Hashimoto [8] Muluh et al. [23]). In fact, an AOE was referred to as problem- difficulty (used inter-changeably in cognitive arithmetic research with problem-size effect) by Ruchkin et al. (1988). Nonetheless, significant amplitude differences at $\mathrm{Pz}$ (higher for division compared to subtraction) in the $500-800 \mathrm{~ms}$ time-window was recorded. Still in this same study, a significant lateralization (C3 versus C4) in amplitude for the division operation was also observed. The complexity of the stimulus (which was acknowledged by the authors themselves) may have been responsible for the anomaly conclusion of AOE in that study.

Parietal dominant P3b has also been linked with brain common response to visual stimuli in MAP [8]. Nonetheless, Kiefer and Dehaene [19] found some PSE in their study. In that study, multiplication of single-digit numbers (between 2 and 9) separated into small (operands ranging from 2 to 5) and large (operands ranging from 6 to 9) size problems presented visually and auditorially in a sequential order multiplication-verification task was presented to their subjects. Operation sign $(\mathrm{x})$ was not employed during problem presentation. Event-related potentials filtered in the $0.15-30 \mathrm{~Hz}$ frequency-band were analyzed for the time course of inferior parietal activation during single-digit multiplication. They obtained significant PSE $\mathrm{x}$ hemisphere interaction in an ascending part of a posterior positivity (possibly P3b) that was maximal at centro-parietal electrodes in a visual modality setting. Amplitude differences in the descending part of this component 
were however not significant in that study. Even though not significant, these authors observed longer latencies for large compared to small size problems. They concluded that the left inferior parietal cortex was active starting about $300 \mathrm{~ms}$ following presentation of the operands of a single-digit multiplication problem. Processing of small size problems was observed to be completed at about $600 \mathrm{~ms}$ but prolonged for large size problems in that study.

Also, Kong et al. [20] reported no amplitude effect of $\mathrm{P} 3 \mathrm{~b}$ as a result of manipulating problem-size at any electrode. However, significant latency differences were obtained at F4 (difficult problems having longer latencies compared to easy ones). Performing out analysis in a Low frequency-band, Iguchi and Hashimoto [8] observed an amplitude enhancement of $\mathrm{P} 3 \mathrm{~b}$ in addition and counting tasks at frontal and temporal regions. They associated this observation with recognition of numeric meaning of presented digits. Parietal dominant P3b was linked with brain common response to visual stimuli and was not affected by any of the tasks condition.

An investigation of processing multiplication was also carried out by Jost et al. [22]. Their subjects multiplied single-digit numbers separated into large size (both operands $>5$ ) and small size (both operands less than or equal to 5 or containing a 2 and 5) in a productionverification condition. The operation sign $(\mathrm{x})$ was employed during problem presentation. Event-related potentials filtered from DC to $40 \mathrm{~Hz}$ were analyzed for facts, rules and strategies usage in single-digit multiplication. Although, these authors noted that the amplitude of this component was not modulated by PSE in their study, the modulation of the amplitude of this component was also suggested to be a measure of cognitive preparation in terms of adjusting attention and processing resources in a multiplication task by same authors. A central and parietal-positivity (corresponding to P3b) has also shown significant $\mathrm{AOE}$ except in the comparisons addition versus subtraction and division versus multiplication in a study by Muluh et al. [23]. Smallsize problems showed significant AOE only in comparisons involving the division operation in that study. Latency modulation by AOE of these components showed greater significance in large-size compared to small-size problems that showed significance only in comparisons involving the division operation.

The amplitude of the N3 component on the other hand has been observed to be significantly modulated by AOE in a few studies. Zhou et al. [15] visually presented (1) addition, (2) multiplication and (3) subtraction of two single digits without the operation sign $(+, \mathrm{x},-)$ to their subjects. Addition and multiplication were further separated into large size addition (sums from 11 to 17 ) and small size multiplication (products ranging from 6 to 24) problems. Event-related potentials were low-pass filtered at $30 \mathrm{~Hz}$ and analysis carried out on the three arithmetic operations. Multiplication was observed to have a higher amplitude compared to addition and subtraction that did not differ with each other at frontal electrode locations. The amplitude effect was attributed to differential retrieval of facts in the different arithmeticoperations by these authors. AOE X PSE interaction also modulated the amplitude of this component in that study (although, this interaction was interpreted as a PSE). These authors however, did not observe any latency effects. In the study of Muluh et al. [23] the amplitude of this component in large-size problems was significantly modulated by arithmetic-operation manipulation when left versus right hemisphere in a frontal-negativity (corresponding to the N3 component) was carried out. Some PSE in frontal negativity activity were also observed by Szu"cs and Cs'epe (2004) in the 380 - 500 ms latency range. For large size problems, a further fronto-parietal negativity appeared between $300-500 \mathrm{~ms}$ relative to small size. A negativity with a maximum at central sites appeared between 380 - $500 \mathrm{~ms}$. The late negativity most probably expresses the enhancement of control and memorization processes in calculating or retrieving larger sums according to these authors.

Zhou et al. [15] recorded some significant AOE in their study. Significant amplitude differences (multiplication higher compared to addition and subtraction that did not differ with each other) at F3, F5, F7, FC3, FC5, FT7, AF3, and C5 between 275 - 334 ms. Latency was not affected at these electrodes. This effect was attributed to differential retrieval of facts in the three operations. Zhou et al. [15] concluded based on source analysis findings that single-digit multiplication involved more phonological processing than single digit addition and subtraction. PSE x AOE interaction was also observed. Significant amplitude differences between large addition and small multiplication occurred over left frontal regions (F1, F3, F5, F7, AF3, AF7, FP1, FC5, FC3) between 296 - $444 \mathrm{~ms}$, this interactive effect was referred to as PSE by these author (i.e., N3 effect was not attributed to PSE in that study). The above findings seem to point to the fact that late ERP components are in general significantly affect by the manipulation of the size or operation during MAP.

\section{Problem-size and arithmetic-operation effects in slow-wave ERP components}

The slow-wave of event-related potentials is composed of a slow frontal negativity and a slow parietal positivity which might be both present or both absent in recordings of EEG data $[29,30]$. Compared to early and late Components, considerable effort have been employed in revealing the PSE and to a lesser extent AOE on amplitudes and latencies of slow-wave components during MAP. Positive and negative slow-wave (pSW, nSW) components - positive and negative peaks occurring after $500 \mathrm{~ms}$ post stimulus presentation are linked with mental processes essential to calculation prior to obtaining the result of arithmetic calculations [10-12,16,17,8,21,13-15,18].

Both pSW and nSW components have been shown to be elicited in all four arithmetic-operations. Previous ERP studies have shown that the anterior positive and posterior negative slow- waves are related to MAP with the "onset" and "offset" of latency, topography and polarity as varying parameters (e.g., Ruchkin et al. [10,11]). However, conflicting observations are also on record [12] showing that the anterior positive slow-waves are associated with calculation but the posterior negative slow-waves are irrelevant to MAP.

Separating ERP components has revealed amplitude enhancement of slow-wave component. For instance, Iguchi and Hashimoto [8] observed amplitude enhancement of a pSW during addition compared to counting digits over frontal, central, parietal and temporal regions with a strong left parietal tendency within $400-820$ ms post-stimulus presentation. Iguchi and Hashimoto in that study also recorded a restricted frontal pSW $820 \mathrm{~ms}$ post-stimulus presentation that was associated with the mental process of handling numerals prior to getting the addition result. The later positivity is similar to that obtained previously by Ruchkin et al. [10-12] in their respective studies. Iguchi and Hashimoto findings seem to suggest the benefits that accrue when the full ERP frequency-band is separated into high and low bands prior to investigating PSE and AOE. They however did not compare low and full frequency bands effects to assess the full benefits of this separation.

$\mathrm{Nu}^{\prime} \mathrm{n}^{\sim} \mathrm{ez}-\mathrm{Pen} \sim a$ and colleagues have also observed significant PSE 
on amplitude at parietal electrodes on the pSW component $[13,14]$. AOE x PSE interaction significantly affected amplitude in addition versus subtraction comparison at parietal electrodes. Although these authors did not explicitly refer to the effect as AOE $\mathrm{x}$ PSE interaction. The amplitude of pSW has recently been observed to be significantly affected by PSE (large problems having larger amplitudes) and training (training reducing the amplitude, i.e., reduces the PSE) as reported by $\mathrm{N}^{\prime} \mathrm{u}^{\sim}$ nez-Pe ${ }^{\sim}$ na [31]. N'u nez-Pe na in 2008 in that study also associated the amplitude effect to direct memory retrieval of arithmetic facts and further speculated that the pSW effects reflected inhibitory processes associated with direct retrieval of answers.

Also, pSW amplitude at frontal electrodes has been linked with the executive function responsible for the allocation of resources and/ or coordination of processes during calculation [10-12]. However, the effect of pSW at centro-posterior electrode locations has been a subject of controversy for decades. For example, no significant amplitude or latency differences have been reported by R"osler and Heil [12]. Pauli et al. [16-18] also observed no amplitude differences on this component but obtained significant latency modulation by PSE. Amplitude effects were interpreted as general information-processing processes that were not specific to MAP in particular by these authors. Latency effects were associated with more time needed in processing large size problems (due to less established problem-to-answer association- pathways in large size problems).

Significant amplitude differences in pSW at parietal electrodes during addition have also been reported by $\mathrm{N}^{\prime} \mathrm{u}^{\sim}$ nez-Pe na et al. [13] A linear trend was observed between increment in addition and voltage amplitude. A significant effect that was associated with problem difficulty in the time windows from $300-800 \mathrm{~ms}$ was observed. Subtraction did not reveal any PSE. Although these authors did not explicitly refer to this effect as AOE, amplitude differences between addition and subtraction were significant at electrode $\mathrm{P} 3$ and $\mathrm{Pz}$ in the $500-700 \mathrm{~ms}$ time-window (higher for subtraction than addition). No effect in the $700-800 \mathrm{~ms}$ time-window. PSE x AOE interaction: Although these authors did not explicitly label the effect PSE x AOE, this interaction modulated the amplitude of the pSW. Operation modulated the increment effect in the $300-400 \mathrm{~ms}$ and $500-600 \mathrm{~ms}$ windows. The larger the increment, the more positive the voltage in addition. Subtraction of 3 or 4 in the $300-800 \mathrm{~ms}$ time-windows was not significant. However, there was a significant difference between these two subtractions and subtracting 2.

$\mathrm{N}^{\prime} \mathrm{u}^{\sim}$ nez-Pe na et al. [14] in their study gave subjects two tasks - adding or subtracting of 2, 4 or 6 to or from the previous number in sequences of five Arabic numerals beginning with even numbers presented successively on the screen. The three increments or reductions were selected so as to manipulate the PSE (2, 3 considered small size addition or subtraction and 4 large size). The fifth number presented completed the sequence either correctly or incorrectly and subjects were required to indicate with finger press of a response button. The operation signs $(+,-)$ were not employed during problem presentation. ERPs were filtered into $0.05-30 \mathrm{~Hz}$ frequency-band. Event-related potentials for the fourth number in the addition and subtraction sequences were analyzed for processing strategies and PSE. These authors reported an amplitude modulation at parietal electrodes which was associated with problem difficulty $(500-800 \mathrm{~ms}$ time-windows) in subtraction and not addition. This was attributed to the fact that addition is used most often and involve direct retrieval compared to subtraction which relies on reconstructive base strategy processing.
Kiefer and Dehaene [19] also recorded a significant PSE in pSW at parietal electrode locations. The amplitude of this component was significantly affected in a posterior positivity that was maximal at centro-parietal electrodes with a greater effect on the left than right hemisphere (higher voltage for small compared to large problems in the visual modality). Latency was not significant but longer for large compared to small size problems at these electrodes (270 - $397 \mathrm{~ms}$ ). Similar results were obtained in the auditory modality. These authors concluded that the left inferior parietal cortex is active starting about $300 \mathrm{~ms}$ following presentation of the operands of a single-digit multiplication problem. Processing of small size problems was observed to be completed at about $600 \mathrm{~ms}$ but prolonged for large size problems in that study. Before then Pauli et al. [17] had shown that the amplitude of pSW was not affect problem-size manipulation. Offset latency was significantly modulated (longer latencies for difficult compared to moderate and easy problems) in that study. This was mainly from easy versus difficult problems. Amplitude of pSW was associated with very general information processing that is not specific to mental arithmetic. Latency effects were associated with more time needed in processing difficult problems. Practice modulated both amplitude and latency.

In a study by Ruchkin et al. [11], subjects performed mentally (1) addition and subtraction by adding the first two-digits of a presented three-digit number followed by subtraction of the third-digit from the sum (considered to be easy), (2) dividing the first two-digits of a presented three-digit number by 7 (considered to be difficult), (3) mentally rotating by determine whether two rotated figures at angles 10 to 20 degrees (considered to be easy) or 150 to 180 degrees(considered to be difficult) were identical or mirror images. The operation signs (+, $\div,-$ ) were not employed during problem presentation. Event-related potentials filtered into $0.03-30 \mathrm{~Hz}$ for the experimental conditions. These authors found a pre-response positivity that was significant at electrode Fpz (higher in difficult tasks compared to easy task) in the 400 - $600 \mathrm{~ms}$ time-windows. This effect was not associated with any single computational stage or stages such as comprehension, calculation and production but rather to executive function which allocates resources and or coordinates a number of processes. This was similar to the previous finding by Ruchkin et al. [10]. They reported a preresponse amplitude of a positivity at electrode Fpz that was significant for conceptual tasks (i.e., higher in division compared to subtraction) in the $2100-2700 \mathrm{~ms}$ time-window. This effect was associated with executive function which allocates resources and or coordinates a number of processing stages in calculation. No significant amplitude no latency differences were observed at the centro-posterior electrode locations.

Negative slow-waves in MAP have enjoyed less "attention" compared to $\mathrm{pSW}$. Nonetheless, AOE x PSE interaction has been shown to significantly affect its amplitude decades ago [10]. Recently, nSW have been associated with MAP involving deliberate calculation procedures [18]. PSE has also been reported to modulate the amplitude of a preresponse-synchronized average nSW. Mean amplitude of the slowwave $100 \mathrm{~ms}$ immediately before reaction time, i.e., time between task and beginning of the response action cursor movement for the choice of answer in those studies [16,17]. Negativity was significantly more at left $(\mathrm{F} 3, \mathrm{C} 3)$ compared to right $(\mathrm{F} 4, \mathrm{C} 4)$ electrodes when problemsize was manipulated in those studies. This effect was associated with mediating strategies (more processing required by large size problems). However, response-synchronized average amplitude differences were not significant in those studies. Late negativity associated with the enhancement of control and memorization processes in calculating 
Citation: Muluh ET (2011) A Review of Event-Related Potential (ERP) Components Employed in Mental Arithmetic Processing Studies. J Neurol Neurophysiol S6. doi:10.4172/2155-9562.S6-001

or retrieving larger sums in addition being maximum at central sites [21] a sustained negativity with an initial phasic maximum at frontal and temporal electrodes and a subsequent gradual increase at central to occipital sites with significant amplitude effects at $\mathrm{Fz}$ and $\mathrm{T} 6$ associated with the recruitment of different neural resources to large and small size problems Jost et al. [16] have also been observed.

Ruchkin et al. [10] also observed amplitude of a late slow negativity at centro-posterior scalp was affected (increase with conceptual difficulty) in the 900 - $1200 \mathrm{~ms}$ time-window. Posterior negativity significantly larger when arithmetic problems were difficult compared to easy ones in the $700-1100 \mathrm{~ms}$ time-window. Amplitude was associated with (1) execution of arithmetic operations and or (2) the amount of information maintained and accessed in short-term memory. These authors concluded that all functional and topographic profile comparisons show that different neural generators underlie late slow- waves. Also reporting on nSW, Jost et al. [22] observed a sustained negativity with an initial phasic maximum at frontal and temporal electrodes and a subsequent gradual increase at central to occipital sites was recorded (higher for large compared to small size problems) in the $1100-2700 \mathrm{~ms}$ time-window. Amplitude was significantly affected at $\mathrm{Fz}$ and $\mathrm{T} 6$ electrodes. This effect was suggested to be a reflection of the recruitment of different neural resources to large and small size problems. A significant amplitude difference at central electrodes and non-significant tendency at parietal electrodes was also recorded (large size problems showed a greater amplitude compared to small size problems in the 630 - $1013 \mathrm{~ms}$ time-window) for nSW by Kiefer and Dehaene [19]. Prior to that, Pauli et al. [17] had observed a $\mathrm{nSW}$ with a pre-response amplitude that was significant at electrode Pz. Negativity was significantly more at left (F3, C3) compared to right (F4, C4) electrodes when difficult and easy problems were compared. This effect was associated with mediating strategies (more processing required by difficult problems).

Beside MAP, increase in amplitude of slow-waves has also been associated with increase in general task demands and/or with behavioral signs of improved processing efficacy [10]. The frontal negativity (FN) has been identified as error-related negativity (ERN) and is associated with error-related processing by some researchers [32-34]. FN is also argued to be related to correct trials processing (also called correct responses negativity (CRN), see studies by Falkenstein et al. [30]; Luu et al. [35]; Batesa et al. [36], Suchan et al. [37]) in-terms of monitoring and evaluation of unfolding action plan and corrective responding. FN has been linked with working memory [38] with its generator source in the anterior cingulate cortex (ACC). FN has also been associated with excitatory preparatory processes, which could be associated with activation of calculation strategies [16]. The work of Bartholow et al. [39] has suggested even revision to models of the effects of conflict on response-related negativity to account for strategic adjustments made in preparation for the response. In conclusion, there is mounting evidence however that the slow parietal positivity is associated with processes associated with mental calculations $[10,8,13-15,11,23]$.

\section{Conclusions}

It is important to note that differences often in experimental design, methodology, manipulation of problem-size, arithmeticoperation, analysis technique(s) among others have been observed to characterize ERP studies investigating PSE, AOE and AOE $\mathrm{x}$ PSE modulation of amplitude and latency in MAP. While it is likely that a number of these differences and others not mentioned may influence modulation of ERP components, a considerable number of them have not been properly resolved. They is mounting evidence from the above reviewed studies indicating that early and late ERP components are involved in mental arithmetic processing at least as indicated by the manipulation of problem-size and arithmetic-operation. Proper experimental conditions that take into account the differences observed in the reviewed studies should lead to more insight into how the brain mentally process arithmetic and information in general.

\section{References}

1. Ashcraft MH (1995) Cognitive psychology and simple arithmetic: A review and summary of new directions. Mathematical Cognition 1: 3-34.

2. Dehaene S (2001) Précis of the Number Sense. Mind \& Language 16: 16-36.

3. Bisanz J, LeFevre J (1992) Understanding Elementary Mathematics. The Nature and Origins of Mathematical Skills. Elsevier Science Publishers, Amsterdam, Netherlands.

4. Ashcraft $\mathrm{MH}$ (1992) Cognitive arithmetic. A review of data theory. Cognition 44 75-106.

5. Zbrodoff NJ, Logan GD (2005) What Everyone Finds: The Problem-Size Effect Handbook of Mathematical Cognition. Psychology Press, New York, USA.

6. Chochon F, Cohen L, van de Moortele PF, Dehaene S (1999) Differentia contributions of the left and right inferior parietal lobules to number processing J Cogn Neurosci 11: 617-630.

7. Delazer M, Girelli L, Granà A, Domahs F (2003) Number processing and calculation--normative data from healthy adults. Clin Neuropsychol 17: 331350 .

8. Iguchi Y, Hashimoto I (2000) Sequential information processing during a mental arithmetic is reflected in the time course of event-related brain potentials. Clin Neurophysiol 111: 204-213.

9. Sandrini M, Miozzo A, Cotelli M, Cappa SF (2003) The residual calculation abilities of a patient with severe aphasia: evidence for a selective deficit of subtraction procedures. Cortex 39: 85-96.

10. Ruchkin DS, Johnson R Jr, Mahaffey D, Sutton S (1988) Toward a functional categorization of slow waves. Psychophysiology 25: 339-353.

11. Ruchkin DS, Johnson R Jr, Canoune H, Ritter W (1991) Event-related potentials during arithmetic and mental rotation. Electroencephalogr Clin Neurophysio 79: 473-487.

12. Rösler F, Heil M (1991) Toward a functional categorization of slow waves: taking into account past and future events. Psychophysiology 28: 344-358.

13. Núñez-Peña MI, Honrubia-Serrano ML, Escera C (2005) Problem size effect in additions and subtractions: an event-related potential study. Neurosci Lett 373 21-25.

14. Núñez-Peña MI, Cortiñas M, Escera C (2006) Problem size effect and processing strategies in mental arithmetic. Neuroreport 17: 357-360.

15. Zhou X, Chen C, Dong Q, Zhang H, Zhou R, et al. (2006) Event-related potentials of single-digit addition, subtraction, and multiplication. Neuropsychologia 44 2500-2507.

16. Pauli P, Lutzenberger W, Rau H, Birbaumer N, Rickard TC, et al. (1994) Brain potentials during mental arithmetic: effects of extensive practice and problem difficulty. Brain Res Cogn Brain Res 2: 21-29.

17. Pauli P, Lutzenberger W, Birbaumer N, Rickard TC, Bourne LE Jr (1996) Neurophysiological correlates of mental arithmetic. Psychophysiology 33: 522 529.

18. Pauli P, Schleichert H, Bourne LE Jr, Birbaumer N (1998) Effects of cortical polarization on mental arithmetic. Brain Res Cogn Brain Res 7: 49-56.

19. Kiefer M, Dehaene S (1997) The time course of parietal activation in single-digit multiplication: Evidence from event-related potentials. Mathematical Cognition 3: $1-30$.

20. Kong J, Wang Y, Shang H, Yang YWAX, Zhuang D (1999) Brain potentials during mental arithmetic - effects of problem difficulty on event-related brain potentials. Neurosci Lett 260: 169-172.

21. Sz"ucs D, Cs'epe V (2004) Access to numerical information is dependent on the modality of stimulus presentation in mental addition: a combined ERP and 
Citation: Muluh ET (2011) A Review of Event-Related Potential (ERP) Components Employed in Mental Arithmetic Processing Studies. J Neurol Neurophysiol S6. doi:10.4172/2155-9562.S6-001

Page 7 of 7

behavioral study. Brain Res Cogn Brain Res19: 10-27.

22. Jost K, Beinhoff U, Hennighausen E, R"osler F (2004a) Facts, rules, and strategies in single-digit multiplication: evidence from event-related brain potentials. Brain Res Cogn Brain Res 20:183-193.

23. Muluh E, Vaughan CL, John LR (2011) High resolution event-related potentials analysis of the arithmetic-operation effect. Clin Neurophysiol 122: 518-529.

24. Zhou X, Chen C, Chen SQC, Chen L (2009) Event-related potentials for simple arithmetic in Arabic digits and Chinese words: a study of the mental representation of arithmetic facts through notation and operation effects. Brain Res1302: 212-224.

25. Jost K, Hennighausen E, R"osler F (2004b) Comparing arithmetic and semantic fact retrieval: Effects of problem size and sentence constraint on event-related brain potentials. Psychophysiology 41: 46-59.

26. De Smedt B, Grabner RH, Studer B (2009) Oscillatory EEG correlates of arithmetic strategy use in addition and subtraction. Exp Brain Res 95: 635-642.

27. Niedeggen M, R"osler F, Jost K (1999) Processing of inconguous menta calculation problems: Evidence for an arithmetic N400 effect. Psychophysiology 36: $307-324$

28. Earle JBB, Garcia-Dergay P, Manniello A, Dowd C (1996) Mathematica cognitive style and arithmetic sign comprehension: A study of EEG alpha and theta activity. Int J Psychophysiol 21: 1-13.

29. Loveless NE, Simpson M, N"a"at"anen R (1987) Frontal negative and parieta positive components of the slow wave dissociated. Psychophysiology 24: 340345

30. Falkenstein M, rg Hoormann J, Christ S, Hohnsbein J (2000) ERP components on reaction errors and their functional significance: a tutorial. Biol Psychol 51: $87-107$.
31. N'ũnez-Pe na Ml (2008) Effect of training on the arithmetic porblem-size effect: an event-related potential study. Exp Brain Res 190: 105-110.

32. Gehring WJ, Goss B, Coles MGH, Meyer DE, Donchin E (1993) A neura system for error detection and compensation. Psychological Science 4: 385390.

33. Bernstein PS, Scheffers MK, Coles MG (1995) Where did I go wrong? A pschophysiological analysis of error detection. J Exp Psychol Hum Percept Perform 21: 1312-1322.

34. Yeung N, Cohen JD, Botvinick MM (2004) The neural basis of error detection: Conflict monitoring and the error-related negativity. Psychol Rev 111: 931-959.

35. Luu P, Flaisch T, Tucker DM (2000) Medial frontal cortex in action monitoring. J Neurosci 20: 464-469.

36. Batesa AT, Kiehlc KA, Laurensa KR, Liddl PF (2002) Error-related negativity and correct response negativity in schizophrenia. Clin Neurophysiol 113: 1454 1463

37. Suchan B, Zoppelt D, Daum I (2003) Frontocentral negativity in electroencephalogram reflects motor response evaluation in humans in correct trials. Neurosci Lett 350: 101-104.

38. Leynes PA, Grey JA, Crawford JT (2006) Event-related potentials ERP evidence for sensorybased action memories. Int J Psychophysiol 62: 193-202.

39. Bartholow BD, Pearson MA, Dickter CL, Sher KJ, Fabiani M, et al. (2005) Strategic control and medial frontal negativity: Beyond errors and response conflict. Psychophysiology 42: 33-42.

This article was originally published in a special issue, Clinical

Neurophysiology: Research and Diagnosis handled by Editor(s). Dr.

Ferdinando Sartucci, Pisa University Medical School, Italy 